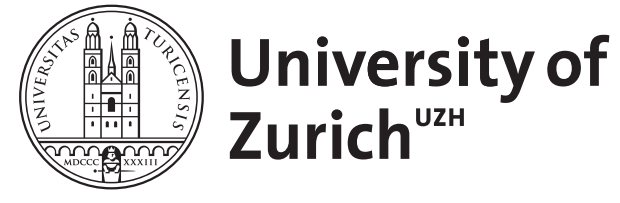

\title{
Konvektiver Transport als möglicher Mechanismus bei Knochenumbauprozessen
}

\author{
Knothe Tate, M L ; Niederer, $\mathrm{P}$; Tepic, S
}

DOI: https://doi.org/10.1515/bmte.1996.41.s1.100

Posted at the Zurich Open Repository and Archive, University of Zurich ZORA URL: https://doi.org/10.5167/uzh-154606

Journal Article

Published Version

Originally published at:

Knothe Tate, M L; Niederer, P; Tepic, S (1996). Konvektiver Transport als möglicher Mechanismus bei Knochenumbauprozessen. Biomedizinische Technik. Biomedical engineering, 41(s1):100-101.

DOI: https://doi.org/10.1515/bmte.1996.41.s1.100 


\title{
Konvektiver Transport als möglicher Mechanismus bei Knochenumbauprozessen
}

\author{
M. L. Knothe Tate ${ }^{1,2}$, P. Niederer ${ }^{1}$, S. Tepic ${ }^{2}$ \\ ${ }^{1}$ Institut für biomedizinische Technik und Medizinische Informatik \\ Universität und ETH Zürich (Schweiz) \\ ${ }^{2} \mathrm{AO}$ Forschungsinstitut, Davos (Schweiz)
}

\section{EINLEITUNG:}

Der Knochen passt sich seiner biologischen und mechanischen Umgebung an, um eine möglichst optimale Form und Struktur für physiologische Belastungen zu erhalten. Trotz aller Forschung ist der genaue Mechanismus dieser Anpassung allerdings noch nicht eindeutig geklärt worden. Die meisten Theorien postulieren einen direkten mechanischen Einfluss auf den Knochenumbau. Eine Voraussetzung dafür wäre, dass jede Zelle des Knochens über eine Art Sensorik verfügen müsste, um Abweichungen vom gewohnten Belastungsmuster zu spüren und sich dementsprechend anpassen zu können; da verschiedene Zellen auch verschieden belastet werden, müsste sogar jede Zelle individuell einprogrammiert sein. Eine andere, eventuell zusätzliche Erklärungsmöglichkeit wäre, die funktionelle Anpassung der Knochenstruktur als Transportproblem zu betrachen. Hierbei müsste der Knochen über keine speziellen Fähigkeiten verfügen, um zu erkennen, ob das normale Spannungsniveau über- oder unterschritten wird, denn die Steuerung des Knochenumbaus wäre nur eine Frage von Angebot und Nachfrage von z.B. Metaboliten und/oder Mineralstoffen.

Obwohl Diffusion ein wichtiger Prozess beim Transport und Austausch von Molekülen im Organismus ist, haben verschiedene Untersuchungen gezeigt, dass in der Knochenmatrix, wo $85 \%$ des Volumes aus lasttragendem Material (Mineral und Collagen) bestehen, Diffusion allein für den molekularen Transport nicht genügen kann, um die Versorgung der Knochenzellen (d.h. Osteozyten) sicherzustellen. Ein weiterer möglicher Mechanismus für den molekularen Transport in der Knochenmatrix ergibt sich aus der Funktion des Knochens im Rahmen des Bewegungsapparates. Wie von Piekarski und Munro 1977 erstmals postuliert [1], führen die aufgrund der zyklischen Belastung des Knochens entstehenden Deformationen hypothetischerweise zu Strömungen der interstitiellen Flüssigkeit im Knochen. Aufgrund dieser belastungsinduzierten Strömungen entstehen konvektive Vorgänge, welche den Transport über grössere Distanzen bewirken könnten.

In dieser Arbeit wird als Teilaspekt der funktionellen Anpassung der Knochenstruktur sowie der metabolischen Anforderungen des Knochens der Einfluss von Diffusions- und Konvektionsvorgängen untersucht. Das Problem wurde mittels theoretischer Finite Elemente
Modellierung und experimentellen ex vivo und in vitro Knochenmodellen (Metacarpus von Schafen) angegangen.

\section{METHODE:}

Theoretische FE Modellierung:

Das Ziel der Modellienung war es, das Verhältnis zwischen konvektivem und diffusivem Transport in einem einfachen Osteon Modell (nach Piekarski und Munro [1]) zu erfassen, und das theoretische Modell mit experimentellen Daten von "daily strain histories" (nach Rubin und Lanyon [2]) zu vergleichen. Zur Modellierung von Mineraltransport wurde die Diffusions-Konvektionsgleichung verwendet. Wegen der extrem langsamen Strömungsgeschwindigkeiten, welche im Knochen vorherrschen, konnten Beschleunigungseffekte vernachlässigt werden. Das Modell wurde mit dem Wärmeleitungspaket von Marc/Mentat ${ }^{\circledR}$ berechnet, da Wärme- und Massentransport sich unter diesen Bedingungen rechnerisch analog verhalten.

Ex Vivo Perfusionsversuche im Schafsmetacarpus:

Es sollten Flüssigkeitsverschiebungen in einem zyklisch belasteten Metacarpus (vom Schaf) in situ gemessen werden. Mit Hilfe einer Infusionspumpe wurde nach Knothe Tate und Knothe [3] ein geschlossener Kreislauf in den Metacarpus in einem ab dem Ellbogen frisch entnommenen Vorderbein aufgebaut. Der Metacarpus wurde mit einer $0.6 \%$ Disulphinblau, $0.1 \%$ Procionrotlösung oder mit einem Bolus Microperoxidase (angepasst nach [4]) während ausgewählten Zeiten perfundiert. Über zwei percutan eingesetzte Schanz'sche Schrauben wurde der Metacarpus während der Perfusion mit einer mechanischen Testmaschine (Instron ${ }^{\text {Q }}$ ) zyklisch belastet, so dass auf der Knochenoberfläche mit aufgeklebten Dehnungsmessstreifen eine Dehnung von maximal 0.2\% gemessen werden konnte. Die kontralaterale Seite wurde gleich perfundiert, es erfolgte aber keine mechanische Belastung. Anzahl Zyklen, Belastungsgrösse und -rate konnten nach vorgegebenen Mustern von Versuch zu Versuch geändert werden, um mögliche Zusammenhänge zwischen Belastungsmuster und Ausmass der Volumenverschiebungen festzustellen. Nach den Versuchen wurden Dünnschnitte des Metacarpus im Querschnitt nach Standardtechniken [4,5] histologisch und histochemisch (im Fall der Microperoxidaseperfusion) aufbereitet und mikro- sowie makroskopisch 
aufgenommen. Die Aufnahmen wurden mit Bildauswertungsprogrammen analysiert und jeweils ein belasteter und ein unbelasteter Metacarpus (von der kontralateralen Seite), bei gleicher Perfusionszeit, miteinander verglichen.

\section{In Vitro Untersuchung der Volumenverschiebungen in Mikroproben:}

Um Flüssigkeitsverschiebungen in einer zyklisch belasteten Mikroprobe des Metacarpus (vom Schaf) zu messen, wurden jeweils zwei zylindrische Proben von Länge $6 \mathrm{~mm}$ und Durchmesser $3 \mathrm{~mm}$ in eine $0.1 \%$ Procionrot Lösung eingelegt. Eine den beiden Proben wurde durch eine speziell für Mikroproben geeignete mechanische Testmaschine (MTS ${ }^{\text {Q }}$ ) zyklisch belastet. Die andere Probe diente als Kontrolle, bei welcher nur Diffusionsvorgänge beim Transport des Markiermittels wirksam waren. Danach wurden die Proben nach Standard histologischen Techniken [5] aufbereitet und unter dem Mikroskop photographisch dokumentiert. Die Aufnahmen wurden mittels Bildverarbeitungstechniken histomorphometrisch untersucht.

\section{ERGEBNISSE:}

Die FE Modellierung hat gezeigt, dass Diffusion allein kaum ausreicht, um genügend Moleküle (Metaboliten, Mineralstoffe) vom Havers'schen Kanal zum äussersten Osteozyten eines Osteons zu transportieren. Belastungsinduzierte Strömungen (Konvektion) verbessern den Transport zwischen dem Havers'schen Kanal und den Osteozyten wesentlich. Für einen ausreichenden Mineralstoff-/Metabolitentransport (kein Knochenabbau) sind vergleichsweise nur wenige konvektive Zyklen pro Tag notwendig $[6,7]$.

Die Resultate des ex vivo Perfusionversuches haben gezeigt, dass bei Perfusionszeiten von 2, 4, 8, 16 und 120 Minuten, die Konzentration von Markiermittel in der Kortikalis des belasteten Knochens entscheidend grösser war als in derjenigen des unbelasteten.

Auch in Untersuchungen der Volumenverschiebungen in Mikroproben von Schafsmetacarpus konnte bewiesen werden, dass die Procionrot Lösung in Mikroproben unter Belastung wesentlich schneller eindringt als in unbelasteten Proben.

\section{DISKUSSION:}

Es wurde mittels FE Methoden gezeigt, dass nur wenige konvektive Zyklen pro Tag notwendig sein könnten, um eine Inaktivitätsatrophie des gesunden Knochens zu verhindern. Dies scheint die Erkenntnisse von Rubin und Lanyon zu bestätigen, nämlich, dass im Minimum 4 Belastungszyklen mit einer Dehnung von $0.2 \%$ bei niedriger Frequenz erforderlich sind, um die normale Knochendichte zu erhalten [2]. Allerdings sind weitere experimentelle Daten unerlässlich für die Validierung des FE-Modelles sowie für die Definition von physiologisch relevanten Randbedingungen und Transportkoeffizienten (u.a. Diffusionskonstanten, Strömungsrate).
Die experimentelle Untersuchung der belastungsinduzierten Strömung im Knochen ist mit praktischen Schwierigkeiten verbunden, da die Lacunae und Canaliculi einen Durchmesser von weniger als $1 \mu \mathrm{m}$ erweisen. Es mussten neue Techniken entwickelt werden, um die Volumenverschiebungen der interstitiellen Flüssigkeit des Knochens messen zu können. Der Schafsmetacarpus wurde aufgrund seiner einfachen Geometrie, seines fast ausschliesslich aus Havers'schen Knochen bestehenden Aufbaus sowie aufgrund seines einfachen vaskulären Systems für die experimentellen Versuche ausgewählt. Die einfache Geometrie erlaubt einen relativ einfachen Vergleich mit physiologischen Belastungsmustern und Computermodellsimulationen. Nicht zuletzt ist der Knochen des Schafes den des Mensches auch physiologisch analog.

Aufgrund des einfachen vaskulären Systems des Metacarpus war es möglich, einen geschlossenen Kreislauf mit sehr kleinen Verlusten aufzubauen; damit hat sich das ex vivo Modell als eine elegante Lösung für die Untersuchung von belastungsinduzierten Flüssigkeitsverschiebungen erwiesen. Die Verknüpfung von aus dem ex vivo Perfusionsmodell gewonnenen Daten mit dem theoretischen FE Modell erfolgt über die Mikroprobenversuche, wobei Diffusionskurven und Konvektionsraten näher untersucht wurden. Weitere Versuchsserien müssen unternommen werden, um Abhängigkeiten zwischen Belastungsmustern (z.B Anzahl Zyklen, Belastungsgrösse und -rate) und Ausmass der Volumenverschiebungen festzustellen.

Ein Ziel dieser experimentellen Arbeiten besteht darin, den Nachweis für die durch theoretische Arbeiten gewonnen Erkenntnisse zu erbringen. Zusätzlich wird das Verständnis über Volumenverschiebungen von interstitieller Flüssigkeit in Kompaktknochen erweitert.

\section{LITERATUR:}

[1] K. Piekarski und M. Munro; Nature 269, 80 (1977)

[2] C. Rubin und L. Lanyon; J Bone Jt Surg 66A, 397 (1984)

[3] M.L. Knothe Tate und U. Knothe; in Vorbereitung (1996)

[4] N. Ayasaka et al.; Arch Oral Biol 37, 363 (1992)

[5] B. Rahn, persönliche Mitteilung

[6] M.L. Knothe Tate, P. Niederer, S. Tepic und S.M. Perren; Combined Orthopaedic Research Societies Meeting, San Diego (1995)

[7] M.L. Knothe Tate, P. Niederer und S. Tepic; 42nd Annual Meeting, Orthopaedic Research Society, Atlanta (1996) 